\title{
Intact Percutaneous Excision (IPEX) for Definitive Diagnosis of High-Risk Breast Lesions
}

\author{
Pat W. Whitworth, $\mathrm{MD}^{1,2}$ \\ ${ }^{1}$ Nashville Breast Center, Nashville, TN; ${ }^{2}$ Vanderbilt University School of Medicine, Nashville, TN
}

\begin{abstract}
Background. Open surgical excision (OSE) is generally recommended when image-guided core needle breast biopsy demonstrates a high-risk lesion (HRL). We evaluated intact percutaneous excision (IPEX) with standard radiologic and histologic criteria for definitive diagnosis of HRL, particularly atypical ductal hyperplasia (ADH).The primary goal was to confirm criteria associated with $<2 \%$ risk for upgrade to carcinoma, equivalent to risk associated with BI-RADS 3 lesions, for which imaging surveillance is considered sufficient.
\end{abstract}

Methods. In an institutional review board-approved prospective trial, 1,170 patients recommended for breast biopsy at 25 institutions received IPEX with a vacuum- and radiofrequency-assisted device. ADH patients in whom the imaged lesion had been removed and the lesion adequately centered for definitive characterization were designated as the potential surgical avoidance population (PSAP) before
OSE. Subsequent OSE specimen pathology was compared with IPEX findings.

Results. In 1,170 patients, 191 carcinomas and 83 (7\%) HRL, including $32 \mathrm{ADH}(3 \%)$, were diagnosed via IPEX. None of the 51 non-ADH HRL were upgraded to carcinoma on OSE $(n=24)$ or, if OSE was declined, on radiologic follow-up $(n=27)$. No ADH lesions meeting PSAP criteria $(n=10)$ were upgraded to carcinoma on OSE; 3 (14\%) of 22 non-PSAP ADH lesions were upgraded to carcinoma on OSE. In summary, no upgrades to carcinoma were made in patients with non-ADH lesions who underwent IPEX or in ADH patients who had IPEX, met histologic and radiologic criteria, and underwent OSE. Conclusions. IPEX combined with straightforward histologic and radiologic criteria and imaging surveillance constitutes acceptable management of image-detected HRL, including ADH.
Electronic supplementary material The online version of this article (doi:10.1245/s10434-011-1961-3) contains supplementary material, which is available to authorized users.

(C) Society of Surgical Oncology 2011

First Received: 30 May 2011;

Published Online: 9 September 2011

P. W. Whitworth, MD

e-mail: patwhitworth@gmail.com 